Pacific Journal of Mathematics

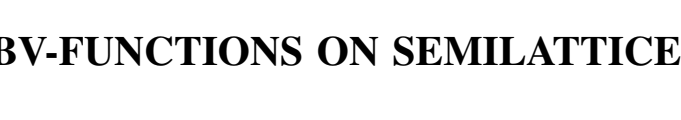




\title{
BV-FUNCTIONS ON SEMILATTICES
}

\author{
J. KIST AND P. H. MASERICK
}

It has been shown that the cone $C$ of completely monotonic functions on a commutative semigroup $G$ with identity induces a vector lattice ordering on the vector space $E=C-C$ spanned by $C$. An intrinsic characterization of the absolute value of the functions in $E$ is desirable. In the present work we offer such a characterization when each member of $G$ is idempotent, i.e. $G$ is a semilattice. A notion of variation and bounded variation (BV) of arbitrary functions on $G$ is introduced. We show that $E$ is precisely the family of $B V$-functions and that if $f \in E$, then our concept of variation of $f$ agrees with the usual absolute value as given by $f \vee(-f)$.

In case the natural order on $G$ is linear, then $C$ is the cone of nonnegative, nondecreasing functions and our notions of variation and bounded variation reduce to the classical concepts. More generally, we show that our variation reduces (not trivially) to the variation defined by Birkhoff [2] for $B V$-valuations on a distributive lattice with largest element.

1. Completely monotonic functions on semilattices. In order to set the stage for our investigations, it will be necessary for us to recall [cf. 1] how the integral representation of a completely monotonic function simplifies when the underlying semigroup is a semilattice.

If $f$ is a real-valued function defined on a commutative semigroup $G$ with identity 1 , then the difference operators $\Delta_{n}$, for $n$ a nonnegative integer, are defined inductively by $\Delta_{0} f(a)=f(a)$, and $\Delta_{n} f\left(a ; b_{1}, \cdots, b_{n}\right)=\Delta_{n-1} f\left(a ; b_{1}, \cdots, b_{n-1}\right)-\Delta_{n-1} f\left(a b_{n} ; b_{1}, \cdots, b_{n-1}\right)$. The function $f$ is said to be completely monotonic if $\Delta_{n} f\left(a ; b_{1}, \cdots, b_{n}\right) \geqq 0$ for all choices of $a, b_{1}, \cdots, b_{n} \in G$. Let $C=C(G)$ denote the family of all completely monotonic functions on $G$ and $C_{1}=\{f \in C: f(1)=1\}$. Then $C$ is a convex cone with base [9] $C_{1}$, in the linear space $R^{G}$ of all real valued functions on $G$. If we equip $R^{G}$ with the topology of pointwise convergence, then the span, $E=C-C$, of $C$ becomes a locally convex linear topological space and $C_{1}$ is compact. From [5] we see that $C_{1}$ is an $r$-simplex, i.e. every $f \in C_{1}$ admits a unique representing measure which is supported by the extremal points (ext $C_{1}$ ) of $C_{1}$, and ext $C_{1}$ is closed. A nontrivial homomorphism from $G$ into the multiplicative semigroup of real numbers in the closed unit interval is called an exponential. The set of exponentials on $G$ 
is denoted by $\exp G$. It is known [cf. 1 or 5] that $\operatorname{ext} C_{1}=\exp G$. It follows that every $f \in E$ admits a unique representing measure $\mu_{f}$ supported by $\exp G$. Thus the map $f \rightarrow \mu_{f}$ is an isomorphism between $E$ and the vector lattice of all regular Borel measures on $\exp (G)$. In particular $E$ is a vector lattice with positive cone $C$.

In the present work we will always assume $G$ is an idempotent semigroup, i.e. a semilattice. If we define $a \leqq b$ whenever $a=a b$ then "ฏ" induces a natural ordering on $G$. A nonvoid subsemigroup $F$ of $G$ whose complement $F^{\prime}$ is an ideal is called a filter, or equivalently $F$, is a nonvoid subsemigroup of $G$ such that $b \in F$ whenever $a \in F$ and $a \leqq b$. A routine check reveals that the exponentials are just the characteristic functions of the filters. Let $\mathscr{F}$ denote the set of all filters in $G$, and $\mathscr{F}(a)=\{F \in \mathscr{F} \mid a \in \mathscr{F}\}$. Then $\mathscr{F}(a b)=\mathscr{F}(a) \cap \mathscr{F}(b)$ (in fact the map $a \rightarrow \mathscr{F}(a)$ is a semilattice isomorphism). The map $\chi_{F} \rightarrow F$ is then a bijection of $\exp G$ onto $\mathscr{F}$. If we impose on $\mathscr{F}$ the topology induced by this latter map then sets of the form $\mathscr{F}(a)$ and $\mathscr{F}(a)^{\prime}$ form a subbase for $\mathscr{F}$. Note $\mathscr{F}$ is a zero dimensional compact Hausdorff space. Let $f \in E$ and $\mu$ be its representing measure. If we transfer $\mu$ to $\mathscr{F}$ then we have

$$
f(a)=\int_{\exp G} \phi(a) d \mu=\mu(\{\dot{\phi} \in \exp G \mid \dot{\phi}(a)=1\})=\mu(\mathscr{F}(a))
$$

and more generally,

$$
\begin{aligned}
\Delta_{n} f\left(a ; b_{1}, \cdots, b_{n}\right) & =\int_{\exp G} \dot{\phi}(a)\left(1-\dot{\phi}\left(b_{1}\right)\right) \cdots\left(1-\dot{\phi}\left(b_{n}\right)\right) d \mu \\
& =\mu\left(\left\{\dot{\phi} \in \exp G \mid \dot{\phi}(a)=1, \dot{\phi}\left(b_{1}\right)=\cdots=\dot{\phi}\left(b_{n}\right)=0\right\}\right) \\
& =\mu\left(\mathscr{F}(a) \cap \mathscr{F}\left(b_{1}\right)^{\prime} \cap \cdots \cap \mathscr{F}\left(b_{n}\right)^{\prime}\right) .
\end{aligned}
$$

This last formula will be used in $\S 2$.

\section{Variation and bounded variation.}

Definition 2.1. (a) The tuples $\left(a ; c_{1}, \cdots, c_{m}\right)$ and $\left(b ; c_{m+1}, \cdots, c_{m+n}\right)$ are said to be related if $c_{i} a b=a b$, i.e., if $c_{i} \geqq a b$ for some $i=$ $1, \cdots, m+n$.

(b) Let $f$ be a real-valued function defined on $G$. The variation $|f|$, and positive variation $f^{+}$of $f$ at a point $a$ in $G$ are defined by

$$
|f|(a)=\sup \sum_{i=1}^{k}\left|\Delta_{n_{i}} f\left(a b_{i, 0} ; b_{i, 1}, \cdots, b_{i, n_{i}}\right)\right|
$$




$$
f^{+}(a)=\sup \sum_{i=1}^{k}\left[\Delta_{n_{i}} f\left(a b_{i, 0} ; b_{i, 1}, \cdots, b_{i, n_{i}}\right)\right] \vee 0,
$$

where the suprema are taken over all finite sets of mutually related tuples $\left\{\left(b_{i, 0} ; b_{i, 1}, \cdots, b_{i, n_{i}}\right)\right\}_{i=1}^{k}$.

(c) The function $f$ defined on $G$ is said to be of bounded variation (a $B V$-function) if $|f|(1)$ is finite.

Observe that for a given family of tuples as in (b), it is possible that $n_{i}=0$ for some $i$ with $1 \leqq i \leqq k$. But since the tuples are pairwise related, we can have $n_{i}=0$ for at most one such index $i$.

The main result of $\S 2$ is Theorem 2.2 where we characterize $E$ as the $B V$-functions on $G$. The following three propositions are necessary for our proof of this theorem.

Proposition 2.2. The tuples $\left(a ; c_{1}, \cdots, c_{m}\right)$ and $\left(b ; c_{m+1}, \cdots, c_{m+n}\right)$ are related if and only if the sets $\mathscr{F}(a) \cap \mathscr{F}\left(c_{1}\right)^{\prime} \cap \cdots \cap \mathscr{T}\left(c_{m}\right)^{\prime}$ and $\mathscr{F}(b) \cap \mathscr{F}\left(c_{m+1}\right)^{\prime} \cap \cdots \cap \mathscr{F}\left(c_{m+n}\right)^{\prime}$ are disjoint.

Proof. Suppose $\mathscr{F}(a b) \cap \mathscr{F}\left(c_{1}\right)^{\prime} \cap \cdots \cap \mathscr{F}\left(c_{m+n}\right)^{\prime}=\phi$. Let $F=$ $\{x \in G: a b \leqq x\}$ then $F$ is a filter on $G, F \in \mathscr{F}(a b)$, and so $F \in \mathscr{F}\left(c_{i}\right)$ for some index $i$. Therefore, $a b \leqq c_{i}$, so the tuples are related.

Conversely, if the tuples are related then $a b \leqq c_{i}$ for some index $i$. Hence if $F$ is a filter on $G$ such that $F \in \mathscr{F}(a b)$, then $F \in \mathscr{F}\left(c_{i}\right)$, i.e., $\mathscr{F}(a b) \cong \mathscr{F}\left(c_{i}\right)$, and so

$$
\mathscr{F}(a b) \cap \mathscr{F}\left(c_{1}\right)^{\prime} \cap \cdots \cap \mathscr{F}\left(c_{m+n}\right)^{\prime}=\phi .
$$

Recall that a semialgebra on a set $X$ is a family $\mathscr{S}$ of subsets of $X$ such that

(i) if $S_{1}$ and $S_{2}$ are members of $\mathscr{S}$, then $S_{1} \cap S_{2}$ is a member of $\mathscr{S}$; (ii) if $S \in \mathscr{S}$, then there is a finite subfamily of pairwise disjoint members of $\mathscr{S}$ whose union is $S^{\prime}$; and (iii) $X \in \mathscr{S}$. An algebra on $X$ is a nonempty family of subsets of $X$ which is closed under the operations of forming finite unions and taking complements. It is easy to verify that the algebra generated by a semialgebra $\mathscr{S}$ consists of those sets which can be expressed as the union of a finite subfamily of pairwise disjoint members of $\mathscr{S}$.

The proof of the next result is straightforward, so will be omitted.

Proposition 2.3. The family $\mathscr{S}$ of sets $\mathscr{F}\left(a_{0}\right) \cap \mathscr{F}\left(a_{1}\right)^{\prime} \cap \cdots \cap$ $\mathscr{F}\left(a_{n}\right)^{\prime}$, where the tuple $\left(a_{0}, \cdots, a_{n}\right)$ ranges over all finite subsets of the semilattice $G$, is a semialgebra on the set $\mathscr{F}$ of all filters on $G$. 
The algebra on $\mathscr{F}$ generated by the semialgebra $\mathscr{S}$ will be denoted by $\mathscr{A}$.

Proposition 2.4. If $f$ is a real-valued function defined on the semilattice $G$, then the function $\mu$ defined by

$$
\mu_{f}\left(\mathscr{F}\left(a_{0}\right) \cap \mathscr{F}\left(a_{1}\right)^{\prime} \cap \cdots \cap \mathscr{F}\left(a_{n}\right)^{\prime}\right)=\Delta_{n} f\left(a_{0} ; a_{1}, \cdots, a_{n}\right)
$$

can be extended to a finitely additive regular set function on $\mathscr{A}$.

Proof. For each element $a$ in $G$, let $\widetilde{a}$ denote the characteristic function of the set $\mathscr{F}(a)$. We claim that the family $\{\widetilde{a}: a \in G\}$ of realvalued functions on $\mathscr{F}$ is linearly independent. To see this, suppose $\alpha_{1} \widetilde{a}_{1}+\cdots+\alpha_{n} \widetilde{a}_{n}=0$, where the $\alpha_{i}$ are real numbers. When given the relative order of $G$, the set $\left\{a_{1}, \cdots, a_{n}\right\}$ must have a maximal element. Assume without loss of generality that $a_{n}$ is such an element, and put $F=\left\{x \in G: a_{n} \leqq x\right\}$. Then $F$ is a filter on $G$ with $a_{n} \in F$, but $a_{i} \in F$ for $i<n$, i.e., $F \in \mathscr{F}\left(a_{n}\right)$, but $F \notin \mathscr{F}\left(a_{i}\right)$ for $i<n$. Therefore, $\left(\alpha_{1} \widetilde{a}_{1}+\cdots+\alpha_{n} \widetilde{a}_{n}\right)(F)=\alpha_{n}=0$. Continuing in this way, we conclude that $\alpha_{1}=\cdots=\alpha_{n}=0$.

We define a linear functional $\bar{f}$ on the linear subspace of $R^{\sigma}$ generated by $\{\widetilde{a}: a \in G\}$ by putting $\bar{f}\left(\sum \alpha_{i} \widetilde{a}_{i}\right)=\sum \alpha_{i} f\left(\alpha_{i}\right)$. Since the family $\{\tilde{a}: a \in G\}$ is linearly independent, and since the mapping $a \rightarrow \widetilde{a}$ is one-to-one, the function $\bar{f}$ is well-defined.

Now let $\left\{\mathscr{F}\left(\alpha_{i, 0}\right) \cap \mathscr{F}\left(\alpha_{i, 1}\right)^{\prime} \cap \cdots \cap \mathscr{F}\left(\alpha_{i, n_{i}}\right)^{\prime}\right\}_{i=1}^{k}$ be a family of pairwise disjoint members of $\mathscr{S}$ whose union is

$$
\mathscr{F}\left(a_{0,0}\right) \cap \mathscr{F}\left(a_{0,1}\right)^{\prime} \cap \cdots \cap \mathscr{F}\left(a_{0, n_{0}}\right)^{\prime} .
$$

The characteristic function of the $i$ th such set, where $0 \leqq i \leqq k$, is

$$
\tilde{a}_{i, 0}\left(1-\tilde{a}_{i, 1}\right) \cdots\left(1-\tilde{a}_{i, n_{i}}\right),
$$

and therefore,

$$
\sum_{1}^{k} \widetilde{a}_{i, 0}\left(1-\widetilde{a}_{i, 1}\right) \cdots\left(1-\widetilde{a}_{i, n_{i}}\right)=\widetilde{a}_{0,0}\left(1-\widetilde{a}_{0,1}\right) \cdots\left(1-\widetilde{a}_{0, n_{0}}\right) \cdot
$$

When the function $\bar{f}$ is applied to both sides of the last equation we obtain

$$
\sum_{1}^{k} \Delta_{n_{i}} f\left(a_{i, 0} ; a_{i, 1}, \cdots, a_{i, n_{i}}\right)=\Delta_{n_{0}} f\left(a_{0,0} ; a_{0,1}, \cdots, a_{0, n_{0}}\right),
$$

and hence 


$$
\begin{aligned}
& \sum_{1}^{k} \mu_{f}\left(\mathscr{F}\left(a_{i, 0}\right) \cap \mathscr{F}\left(a_{i, 1}\right)^{\prime} \cap \cdots \cap \mathscr{F}\left(a_{i, n_{i}}\right)^{\prime}\right) \\
= & \mu_{f}\left(\mathscr{F}\left(a_{0,0}\right) \cap \mathscr{F}\left(a_{0,1}\right)^{\prime} \cap \cdots \cap \mathscr{F}\left(a_{0, n_{0}}\right)^{\prime}\right) .
\end{aligned}
$$

It follows that the set function $\mu_{f}$ on $\mathscr{S}$ has a well-defined and unique finitely additive extension to $\mathscr{A}$. This extension is also denoted by $\mu_{f}$.

The regularity of $\mu_{f}$ on $\mathscr{A}$ is a consequence of the fact that the elements of the semialgebra $\mathscr{S}$ and hence the elements of the algebra $\mathscr{A}$ are open-and-closed.

The following is the main result of the section. The symbol $|\nu|$ is used to denote the variation of the measure $\nu$ [4]. Recall that $E$ is the vector lattice spanned by the completely monotonic functions.

THEOREM 2.5. Let $f$ be a real valued function defined on the semilattice $G$ with identity. Then $f$ is a member of $E$ if and only if it is of bounded variation on $G$. Moreover, if $f$ is a $B V$-function and if $\nu_{f}$ is the regular Borel measure which represents $f$, then

$$
\begin{aligned}
|f|(a) & =\left|\nu_{f}\right|(\mathscr{F}(a)), \\
f^{+}(a) & =\nu_{f^{+}}(\mathscr{F}(a)) \text { for each } a \in G .
\end{aligned}
$$

Finally $|f|=f \vee(-f)$ and $f^{+}=f \vee 0$.

Proof. For each $f \in C$, let $\mu_{f}$ be the finitely additive set function on $\mathscr{A}$ defined in Proposition 2.4 by

$$
\mu_{f}\left(\mathscr{F}(a) \cap \mathscr{F}\left(b_{1}\right)^{\prime} \cap \cdots \cap \mathscr{F}\left(b_{n}\right)^{\prime}\right)=\Delta_{n} f\left(a ; b_{1}, \cdots, b_{n}\right)
$$

then the map $f \rightarrow \mu_{f}$ is an isomorphism of the cone $C$ onto the cone of all nonnegative finitely additive set functions on $\mathscr{A}$. This map therefore admits a unique extension to an isomorphism of $E$ onto the vector lattice of all finitely additive $B V$-set functions on $\mathscr{A}$. Therefore, this extension is order preserving and necessarily assumes the form (i). The definition of variation of a set function [4], Proposition 2.2 and (i) above imply:

$$
\begin{aligned}
(f \vee-f)(a) & =\left|\mu_{f}\right|(\mathscr{F}(a)) \\
& =\sup \sum\left|\mu_{f}\left(\mathscr{F}\left(a b_{i, 0}\right) \cap \mathscr{F}\left(b_{i, 1}\right)^{\prime} \cap \cdots \cap \mathscr{F}\left(b_{i, n_{i}}\right)^{\prime}\right)\right| \\
& =\sup \sum\left|\Delta_{n_{i}} f\left(a b_{i, 0} ; b_{i, 1}, \cdots, b_{i, n_{i}}\right)\right| \\
& =|f|(a)
\end{aligned}
$$

where the first supremum is taken over all finite collections of mutually disjoint members of $\mathscr{A}$ and the second is taken over all finite collections of mutually related tuples. Since a similar argument shows that 


$$
(f \vee 0)(a)=f^{+}(a)
$$

for all $f \in E$, the first and last assertions of the theorem are established.

To establish the intermediate formulas, let $\mu$ be a nonnegative finitely additive set function on $\mathscr{A}$ and let $f$ be defined by $f(\alpha)=$ $\mu(\mathscr{F}(a))$. Then $f \in C$, so there exists a unique nonnegative regular Borel measure $\nu$, such that $\nu$ represents $f$. It follows that $\nu$ is an extension, and in fact the only extension, of $\mu$. Thus the map $\mu \rightarrow \nu$ defines an isomorphism of the cone of finitely additive set functions on $\mathscr{A}$ onto the nonnegative regular Borel measures. It now follows that every finitely additive $B V$-measure $\mu$ admits a unique extension $\nu$, namely the unique representing measure for the $B V$-function $f$ defined by $f(a)=\mu(\mathscr{F}(a))$. Since this extension is order preserving we must have $|\mu|(A)=|\nu|(A)$ and $\mu^{+}(A)=\nu^{+}(A)$ for all $A \in \mathscr{A}$. The desired formulas now follow from (ii) and (iii).

3. Valuations, Recall [2] that a valuation on a lattice $L=$ $(L ; \bigvee, \Lambda)$ is a real-valued function $f$ defined on $L$ which satisfies the identity

$$
f(a)+f(b)=f(a \vee b)+f(a \wedge b) .
$$

Now assume that $L$ is a distributive lattice with largest element 1. Denote by $V=V(L)$ the set of all nonnegative, nondecreasing valuations on $L$ and let $V_{1}=\{f \in V: f(1)=1\}$.

Throughout this section, the symbol $G$ will denote the semilattice $(L ; \Lambda)$, and $C=C(G)$ will denote the cone of all completely monotonic functions defined on $G$.

Proposition 3.1. If $f$ is a valuation on a distributive lattice $L$, then

$$
\Delta_{n} f\left(a ; b_{1}, \cdots, b_{n}\right)=\Delta_{1} f\left(a ; b_{1} \vee \cdots \vee b_{n}\right)
$$

for each integer $n \geqq 1$.

Proof. The assertion is obviously true if $n=1$. If $n=2$, then

$$
\begin{aligned}
\Delta_{2} f\left(a ; b_{1}, b_{2}\right) & =f(a)-f\left(a \wedge b_{1}\right)-f\left(a \wedge b_{2}\right)+f\left(a \wedge b_{1} \wedge b_{2}\right) \\
& =f(a)-f\left(\left(a \wedge b_{1}\right) \vee\left(a \wedge b_{2}\right)\right) \\
& =f(a)-f\left(a \wedge\left(b_{1} \vee b_{2}\right)\right) \\
& =\Delta_{1} f\left(a ; b_{1} \vee b_{2}\right) .
\end{aligned}
$$

Assume that the result is true for $n=k \geqq 2$; then 


$$
\begin{aligned}
\Delta_{k+1} f\left(a ; b_{1}, \cdots, b_{k+1}\right) & =\Delta_{k} f\left(a ; b_{1}, \cdots, b_{k}\right)-\Delta_{k} f\left(a \wedge b_{k+1} ; b_{1}, \cdots, b_{k}\right) \\
& =\Delta_{1} f\left(a ; b_{1} \vee \cdots \vee b_{k}\right)-\Delta_{1}\left(a \wedge b_{k+1} ; b_{1} \vee \cdots \vee b_{k}\right) \\
& =\Delta_{2} f\left(a ; b_{1} \vee \cdots \vee b_{k}, b_{k+1}\right) \\
& =\Delta_{1} f\left(a ; b_{1} \vee \cdots \vee b_{k+1}\right) .
\end{aligned}
$$

Proposition 3.2. The set $V$ is a closed extremal [4] subset of $C$.

Proof. It is immediate that $V_{1}$ is closed in $C_{1}$. (Recall that $C_{1}$ carries the topology of pointwise convergence.)

Note that if $f$ is an element of $C$, then $0 \leqq \Delta_{2} f(a \vee b ; a, b)=$ $f(a \vee b)-f(a)-f(b)+f(a \wedge b)$ and so $f(a)+f(b) \leqq f(a \vee b)+f(a \wedge b)$ for each pair of elements $a, b$ in $L$.

Now let $f$ be a valuation on $L$, and suppose that $f=\alpha f_{1}+(1-\alpha) f_{2}$ with $f_{1}$ and $f_{2}$ elements of $C$, and $0<\alpha<1$. If for some pair $a, b$ in $L$ we have $f_{j}(a)+f_{j}(b)<f_{j}(a \vee b)+f_{j}(a \wedge b)$ for $j=1$ or 2 , then

$$
\begin{aligned}
f(a)+f(b) & =\alpha f_{1}(a)+(1-\alpha) f_{2}(a)+\alpha f_{1}(b)+(1-\alpha) f_{2}(b) \\
& <\alpha\left[f_{1}(a \vee b)+f_{1}(a \wedge b)\right]+(1-\alpha)\left[f_{2}(a \vee b)+f_{2}(a \wedge b)\right] \\
& =f(a \vee b)+f(a \wedge b),
\end{aligned}
$$

which is a contradiction. Thus, the cone $V$ is an extremal subset of the cone $C$.

In particular this implies that the vector space $V-V$ is a sublattice of $E=C-C$. Thus by Theorem 2.5, if $f \in V$ then, the absolute value of $f$ with respect to the vector lattice $V-V$ agrees with the variation $|f|$ of $f$ defined in 2.1. In summary we may state:

THEOREM 3.3. The vector space $V-V$ is a vector lattice which consists of all valuations of bounded variation. Its positive cone is the cone of all nonnegative nondecreasing valuations on $L$ and $|f|=$ $f \vee(-f)$ for all $f \in V-V$.

From Proposition 3.1, if $f$ is a valuation and $a \in L$ then

$$
|f|(a)=\sup \sum_{i=1}^{k}\left|\Delta_{1} f\left(a \wedge b_{i} ; c_{i}\right)\right|+|f(a \wedge b)|
$$

where the arguments of the summands range over all finite sets of mutually related pairs $\left\{a \wedge\left(b_{i} ; c_{i}\right)\right\}_{i}$ such that $c_{i} \wedge\left(a \wedge b \wedge b_{i}\right)=$ $a \wedge b \wedge b_{i}$.

It is not hard to see that this notion of variation agrees with the classical concept of variation of real valued functions on linearly ordered lattices. Birkhoff [2] has also generalized the classical 
concept of variation to valuations on a lattice. We will distinguish his definition from ours by referring to his as linear variation.

DeFinition 3.4. (Birkhoff [2]). Let $f$ be a valuation on a distributive lattice $L$. Then the linear variation $|f|_{0}$ is defined by

$$
|f|_{0}(a)=\sup \sum_{i=1}^{n-1}\left|f\left(x_{i+1}\right)-f\left(x_{i}\right)\right|+\left|f\left(x_{1}\right)\right|
$$

and the linear positive variation $(f)_{0}^{+}$is defined by

$$
(f)_{0}^{+}=\sup \sum_{i=1}^{n-1}\left[f\left(x_{i+1}\right)-f\left(x_{i}\right)\right] \vee 0+\left[f\left(x_{1}\right)\right] \vee 0 ;
$$

where the suprema are taken over all ascending chains $x_{1} \leqq x_{2} \leqq \cdots \leqq$ $x_{n}=a$.

In [2], using methods different from ours, Birkhoff essentially shows that Theorem 3.3 is valid when "variation" is replaced by linear variation." Hence it follows that $|f|=|f|_{0}$ whenever either $|f|(1)<\infty$ or $|f|_{0}(1)<\infty$. We will now prove a series of algebraic lemmas which will lead to an alternate proof of this fact; in this approach we use Theorem 3.3 rather than Birkhoff's results.

Lemma 3.5. If $f$ is a valuation on $L$ then $|f|_{0} \leqq|f|$.

Proof. Let $x_{1} \leqq x_{2} \leqq \cdots \leqq x_{n}=a$ be a chain in $L$. Then

$$
\sum_{i=1}^{n-1}\left|f\left(x_{i+1}\right)-f\left(x_{i}\right)\right|+\left|f\left(x_{1}\right)\right| \leqq \sum_{i=1}^{n-1}\left|\Delta_{1} f\left(x_{i+1} ; x_{i}\right)\right|+\left|f\left(x_{1}\right)\right| .
$$

If $n+1>i>j$ then $x_{i} \geqq x_{j}=x_{i+1} \wedge x_{j}$ so that $\left\{\left(x_{i+1} ; x_{i}\right) \mid i=1,2, \cdots, n\right\}$ is a mutually related set of ordered pairs.

For the remainder of this section we find it convenient to let $N(m, n)(n \geqq m)$ be the class of all strictly increasing functions $\alpha \mid\{1,2, \cdots, m\} \rightarrow\{1,2, \cdots, n\}$.

LEMmA 3.6. If $f$ is a valuation on $L$ and $a_{1}, a_{2}, \cdots, a_{k+1} \in L$ then for a fixed $n: 0 \leqq n<k$

$$
\begin{aligned}
f & {\left[\mathrm{~V}\left\{\left(a_{\alpha(1)} \wedge a_{\alpha(2)} \cdots \wedge a_{\alpha(n+1)}\right) \mid \alpha \in N(n+1, k)\right\}\right] } \\
& +f\left[\bigvee\left\{\left(a_{\alpha(1)} \wedge a_{\alpha(2)} \cdots \wedge a_{\alpha(n)} \wedge a_{k+1}\right) \mid \alpha \in N(n, k)\right\}\right] \\
=f & {\left[\bigvee\left\{\left(a_{\alpha(1)} \wedge a_{\alpha(2)} \cdots \wedge a_{\alpha(n+1)}\right) \mid \alpha \in N(n+1, k+1)\right\}\right] } \\
& +f\left[\bigvee\left\{\left(a_{\alpha(1)} \wedge a_{\alpha(2)} \cdots \wedge a_{\alpha(n+1)} \wedge a_{k+1} \mid \alpha \in N(n+1, k)\right\}\right] .\right.
\end{aligned}
$$

Proof. If we let $a$ and $b$ the respective arguments of $f$ in the first and second terms to the left of the equality, then, 


$$
a \bigvee b=\bigvee\left\{a_{\alpha(1)} \wedge a_{\alpha(2)} \cdots \wedge a_{\alpha(n+1)} \mid \alpha \in N(n+1, k+1)\right\}
$$

Moreover, the generalized distributive law implies,

$$
\begin{gathered}
a \wedge b=\bigvee\left\{\left(a_{\alpha(1)} \wedge a_{\alpha(2)} \cdots \wedge a_{\alpha(n+1)} \wedge a_{\beta(1)} \wedge a_{\beta(2)} \cdots \wedge a_{\beta(n)} \wedge a_{k+1}\right)\right. \\
\mid \alpha \in N(n+1, k), \beta \in N(n, k)\}
\end{gathered}
$$

so that

$$
\left.a \wedge b=\mathrm{V}\left\{a_{\alpha(1)} \wedge a_{\alpha(2)} \cdots \wedge a_{\alpha(n+1)} \wedge a_{k+1}\right) \mid \alpha \in N(n+1, k)\right\} .
$$

The assertion now follows since $f(a)+f(b)=f(a \vee b)+f(a \wedge b)$.

Lemma 3.7. If $f$ is valuation on $L$ and if $a_{1}, a_{2}, \cdots, a_{k} \in L$ then

$$
\left.\sum_{i=1}^{k} f\left(a_{i}\right)=\sum_{i=1}^{k} f\left[\mathbf{V}\left\{a_{\alpha(1)} \wedge a_{\alpha(2)} \cdots \wedge a_{\alpha(i)}\right) \mid \alpha \in N(i, k)\right\}\right] .
$$

Proof. The assertion is clearly valid if $k=1$. If we assume its validity for $k$ then

$$
\begin{aligned}
\sum_{i=1}^{k+1} f\left(a_{i}\right)= & \sum_{i=1}^{k} f\left(a_{i}\right)+f\left(a_{k+1}\right) \\
= & \sum_{i=1}^{k} f\left[\bigvee\left\{a_{\alpha(1)} \wedge a_{\alpha(2)} \cdots \wedge a_{\alpha(i)} \mid \alpha \in N(i, k)\right\}\right]+f\left(a_{k+1}\right) \\
= & f\left[\bigvee_{i=1}^{k} a_{i}\right]+f\left(a_{k+1}\right) \\
& +\sum_{i=2}^{k} f\left[\mathbf{V}\left\{a_{\alpha(1)} \wedge a_{\alpha(2)} \cdots \wedge a_{\alpha(i)} \mid \alpha \in N(i, k)\right\}\right] .
\end{aligned}
$$

Applying Lemma 3.6 to the first term of the above expression with $n=0$ and $k=1$ yields

$$
\begin{aligned}
\sum_{i=1}^{k+1} f\left(a_{i}\right)= & f\left[\bigvee_{i=1}^{k+1} a_{i}\right]+f\left[\bigvee_{i=1}^{k}\left(a_{i} \wedge a_{k+1}\right)\right] \\
& +\sum_{i=2}^{k} f\left[\bigvee\left\{a_{\alpha(1)} \wedge a_{\alpha(2)} \cdots \wedge a_{\alpha(i)} \mid \alpha \in N(i, k)\right\}\right] .
\end{aligned}
$$

Reapplying Lemma 3.6 to the second term to the right of equality and the first term of the summation with $n=1$ and $k=k$ yields

$$
\begin{aligned}
\sum_{i=1}^{k+1} f\left(a_{i}\right)= & f\left[\bigvee_{i=1}^{k+1} a_{i}\right]+f\left[\mathbf{V}\left\{\left(a_{\alpha(1)} \wedge a_{\alpha(2)} \mid \alpha \in N(2, k+1)\right\}\right]\right. \\
& +f\left[\bigvee\left\{\left(a_{\alpha(1)} \wedge a_{\alpha(2)} \wedge a_{k+1} \mid \alpha \in N(2, k)\right\}\right]\right. \\
& +\sum_{i=3}^{k} f\left[\mathbf{V}\left\{\left(a_{\alpha(1)} \wedge a_{\alpha(2)} \cdots a_{\alpha(i)} \mid \alpha \in N(i, k)\right\}\right]\right.
\end{aligned}
$$

Successively repeating this process proves the assertion for $k+1$. 
LEMMA 3.8. If $\left\{\left(a_{i}, b_{i}\right) \mid i=1,2, \cdots, k\right\}$ and $\left(a_{k+1}\right)$ are $k+1$ mutually related tuples such that $b_{i} \leqq a_{i}$ for each $i=1,2, \cdots, k$ then

$$
\begin{aligned}
& \mathrm{V}\left\{\left(b_{\alpha(1)} \wedge b_{\alpha(2)} \cdots \wedge b_{\alpha(n)} \mid \alpha \in N(n, k)\right\}\right. \\
\geqq & \mathbf{V}\left\{\left(a_{\alpha(1)} \wedge a_{\alpha(2)} \cdots \wedge a_{\alpha(n+1)} \mid \alpha \in N(n+1, k+1)\right\}\right.
\end{aligned}
$$

Proof. Let $a=\left(a_{\alpha(1)} \wedge a_{\alpha(2)} \cdots \wedge a_{\alpha(n+1)}\right)$ for some $\alpha \in N(n+1, k+1)$.

Case 1. $\alpha(n+1) \neq k+1$. We may assume the existence of $j(j=1,2, \cdots, n)$ such that $b_{\alpha(j)} \geqq a$. Then $b_{\alpha(j)} \geqq a_{\alpha(j)} \wedge a_{\alpha(l)} \geqq a$ for any $l=1,2, \cdots, n+1$. Therefore if $j \neq l$ the mutual relatedness implies

$$
b_{\alpha(l)} \geqq a_{\alpha(j)} \wedge a_{\alpha(l)} \geqq a \text {, so that } \bigwedge_{l \neq j} b_{\alpha(l)} \geqq a .
$$

Case 2. $\alpha(n+1)=k+1$. Then by mutual relatedness,

$$
b_{\alpha(j)} \geqq a_{\alpha(n+1)} \wedge a_{\alpha(j)} \geqq a \text {, so that } \bigwedge_{j=1}^{n} b_{\alpha(j)} \geqq a .
$$

The assertion easily follows from these two cases.

LEMMA 3.9. If $f$ is a valuation on $L$ then

$$
\left(f_{0}^{+}\right) \geqq f^{+} \text {. }
$$

Proof. Consider the sum $\sum_{i=1}^{k} \Delta_{1} f\left(a_{i} ; b_{i}\right)+f\left(a_{k+1}\right)$.

Without loss of generality we make the following assumptions:

(i) Each term in the summand is strictly positive.

(ii) $b_{i}<a_{i} \leqq a$ for each $i=1,2, \cdots, k$ and $a_{k+1} \leqq a$.

(iii) The set $\left\{\left(a_{i} ; b_{i}\right) \mid i=1,2, \cdots, k\right\} \cup\left\{a_{k+1}\right\}$ is mutually related.

From Lemma 3.7 we have

$$
\begin{aligned}
& \sum_{i=1}^{k} \Delta_{1} f\left(a_{i} ; b_{i}\right)+f\left(a_{k+1}\right) \\
= & \sum_{i=1}^{k}\left(f\left[\mathrm{~V}\left\{\left(a_{\alpha(1)} \wedge a_{\alpha(2)} \cdots \wedge a_{\alpha(i)}\right) \mid \alpha \in N(i, k+1)\right\}\right]\right. \\
& -f\left[\mathrm{~V}\left\{\left(b_{\alpha(1)} \wedge b_{\alpha(2)} \cdots \wedge b_{\alpha(i)} \mid \alpha \in N(i, k)\right\}\right]\right) \\
& +f\left(a_{1} \wedge a_{2} \cdots \wedge a_{k+1}\right) .
\end{aligned}
$$

Lemma 3.8 and assumption (ii) above imply that the arguments of the terms in the above expression form a decreasing sequence. The assertion easily follows.

THEOREM 3.10. If $f$ is a valuation on a distributive lattice $L$ then $f^{+}=(f)_{0}^{+}$. Moreover $f$ is of bounded variation $(|f|<\infty)$ if and only if $f$ is of bounded linear variation $\left(|f|_{0}<\infty\right)$, in this case, $|f|=|f|_{0}$. 
Proof. The first assertion follows from Lemmas 3.5 and 3.9. Lemma 3.5 also implies that $|f|_{0}<\infty$ whenever $|f|<\infty$. Conversely if $f$ is of bounded linear variation then $(f)_{0}^{+}(1)<\infty$ and $(-f)_{0}^{+}(1)<\infty$ so that

$$
|f|(1) \leqq f^{+}(1)+(-f)^{+}(1) \leqq(f)_{0}^{+}(1)+(-f)_{0}^{+}(1)<\infty .
$$

In view of Lemma 3.5, to complete the proof we need only verify that $|f| \leqq|f|_{0}$ if $|f|_{0}<\infty$. Given $\varepsilon>0$, there exists a chain $x_{1}<x_{2}<\cdots<x_{n}=a$ in $L$ such that

$$
(f)_{0}^{+}(a) \leqq \sum_{i=1}^{n-1}\left[f\left(x_{i+1}\right)-f\left(x_{i}\right)\right] \vee 0+f\left(x_{1}\right) \vee 0+\varepsilon / 2 .
$$

But it follows from Theorem 3.3 that $|f|(\alpha)=2 f^{+}(\alpha)-f(a)$. Therefore

$$
\begin{aligned}
|f|(a)= & 2(f)_{0}^{+}(a)-f(a) \leqq 2 \sum_{i=1}^{n-1}\left[f\left(x_{i+1}\right)-f\left(x_{i}\right)\right] \vee 0 \\
& +2\left(f\left(x_{1}\right) \vee 0\right)-f(a)+\varepsilon \\
= & 2 \sum_{i=1}^{n-1}\left[f\left(x_{i+1}\right)-f\left(x_{i}\right)\right] \vee 0+2\left(f\left(x_{1}\right) \vee 0\right) \\
& \quad-\sum_{i=1}^{n-1}\left[f\left(x_{i+1}\right)-f\left(x_{i}\right)\right] f\left(x_{1}\right)+\varepsilon \\
= & \sum_{i=1}^{n}\left|f\left(x_{i+1}\right)-f\left(x_{i}\right)\right|+\left|f\left(x_{1}\right)\right|+\varepsilon \\
\leqq & |f|_{0}+\varepsilon . \text { Hence }|f| \leqq|f|_{0} .
\end{aligned}
$$

Let $f \in V-V$. We turn our attention to constructing the representing measure for $f$. To this end we will define a filter on $G$ to be prime if $a \vee b \in G$ implies either $a \in G$ or $b \in G$. The following proposition is essentially contained in [3].

Proposition 3.11. A function $f \in V_{1}$ is an extreme point of $V_{1}$ if and only if it is the characteristic function of a prime filter (i.e. a zero-one lattice homomorphism).

Proof. A routine check reveals that the zero-one lattice homomorphisms agree with the characteristic functions of the prime filters. Clearly they are all extremal elements of $V$. To prove the converse, observe that Lemma 3.2 implies that $V_{1}$ is an extremal subset of $C_{1}$. The assertion follows since each extremal point must be an exponential (or the characteristic function of a filter) as well as a valuation.

If we let $P$ denote the set of all prime filters and $P(\alpha)=P \cap F(\alpha)$ then $\mathscr{P}(1)$ is a compact zero dimensional subspace of $\mathscr{F}(1)$. But if $f \in V-V$ then its representing measure is supported by the extreme points of $V_{1}$, or in our setting, by $\mathscr{P}(1)$. Therefore Theorem 2.5 
implies the first part of the following.

THEOREM 3.12. If $f$ is a valuation of bounded variation, then there exists a unique regular Borel measure $\nu_{f}$ on $\mathscr{P}(1)$ such that

$$
\begin{aligned}
& |f|(a)=\left|\nu_{f}\right|(\mathscr{P}(a)) \\
& f^{+}(a)=\nu_{f}^{+}(\mathscr{P}(a)) .
\end{aligned}
$$

Moreover the map $a \rightarrow \mathscr{P}(a)$ is a lattice isomorphism while the map $f \rightarrow \nu_{f}$ in a vector lattice isomorphism.

Proof. The only assertion which we have not proved is that the map $a \rightarrow \mathscr{P}(a)$ is biunique. But this is a known result from lattice theory. In fact it can be shown that every filter is the intersection of prime filters.

Acknowledgements. The results of this paper are an outgrowth of a seminar which was conducted by the second named author at the Pennsylvania State University in 1967. The authors wish to thank Professors F. R. Deutsch, N. J. Fine and P. D. Morris who participated and offered valuable suggestions.

It has come to our attention that S. E. Newman has recently announced results [7], [8] which are related to the work in $\S 2$. In particular, using combinatorial technique, he has introduced a formally different notion of $B V$-functions on a semilattice. His emphasis is not, as is ours, on the lattice theoretic properties of the $B V$ functions (eg. he has not announced a concept of variation as such) but rather on the multiplicative structure of these functions. Some of his work carries over to our setting quite naturally. For example his theorem that the product of two $B V$-functions is again a $B V$ function follows for $B V$-functions in our sense from [5, Cor. 1.6].

\section{REFERENCES}

1. H. Bauer, Konvexitat in Topologischen Vehtorräumen, Lecture notes University of Hamburg 1963/64.

2. G. Birkhoff, Latiice Theory, Amer. Math. Soc., Colloquium Publication 25, New York (1948).

3. G. Choquet, Theory of capacities, Ann. Inst. Fourier (Grenoble) 5 (1954), 131-295.

4. N. Dunford and J. T. Schwartz, Linear Operators, Part I, Interscience, New YorkLondon, 1958.

5. N. Fine and P. H. Maserick, On the simplex of completely monotonic functions on a commutative semigroup, Canad. J. Math., XXII (1970), 317-326.

6. J. Kist and P. H. Maserick, Functions of bounded variation on a commutative idempotent semigroup, Abstract 653-29, Notices of Amer. Math. Soc., 15 (1968). 
7. S. E. Newman, Functions of bounded variation on idempotent semigroups, Abstract 663-458, Notices of Amer. Mach. Soc., 16 (1969).

8. — Measure algebras and functions of bounded variation on idempotent semigroups, Bull. Amer. Math. Soc., 75 (1969), 1396-1400.

9. R. R. Phelps, Lectures on Choquet Theory, van Nostrand, Toronto-New YorkLondon 1966.

Received June 15, 1970 and in revised form February 11, 1971.

Pennsylvania State University 



\title{
PACIFIC JOURNAL OF MATHEMATICS
}

\author{
EDITORS
}

\author{
H. SAMelson \\ Stanford University \\ Stanford, California 94305 \\ C. R. HoBBy \\ University of Washington \\ Seattle, Washington 98105
}

J. DugunduI

Department of Mathematics

University of Southern California

Los Angeles, California 90007

RICHARD ARENS

University of California

Los Angeles, California 90024

\section{ASSOCIATE EDITORS}

\author{
E. F. BeCKENBACH
}

B. H. NEUMANN

F. WoLF

K. YOSHIDA

\section{SUPPORTING INSTITUTIONS}

\author{
UNIVERSITY OF BRITISH COLUMBIA \\ CALIFORNIA INSTITUTE OF TECHNOLOGY \\ UNIVERSITY OF CALIFORNIA \\ MONTANA STATE UNIVERSITY \\ UNIVERSITY OF NEVADA \\ NEW MEXICO STATE UNIVERSITY \\ OREGON STATE UNIVERSITY \\ UNIVERSITY OF OREGON \\ OSAKA UNIVERSITY \\ UNIVERSITY OF SOUTHERN CALIFORNIA
}

\author{
STANFORD UNIVERSITY \\ UNIVERSITY OF TOKYO \\ UNIVERSITY OF UTAH \\ WASHINGTON STATE UNIVERSITY \\ UNIVERSITY OF WASHINGTON \\ AMERICAN MATHEMATICAL SOCIETY \\ CHEVRON RESEARCH CORPORATION \\ NAVAL WEAPONS CENTER
}

The Supporting Institutions listed above contribute to the cost of publication of this Journal, but they are not owners or publishers and have no responsibility for its content or policies.

Mathematical papers intended for publication in the Pacific Journal of Mathematics should be in typed form or offset-reproduced, (not dittoed), double spaced with large margins. Underline Greek letters in red, German in green, and script in blue. The first paragraph or two must be capable of being used separately as a synopsis of the entire paper. The editorial "we" must not be used in the synopsis, and items of the bibliography should not be cited there unless absolutely necessary, in which case they must be identified by author and Journal, rather than by item number. Manuscripts, in duplicate if possible, may be sent to any one of the four editors. Please classify according to the scheme of Math. Rev. Index to Vol. 39. All other communications to the editors should be addressed to the managing editor, Richard Arens, University of California, Los Angeles, California, 90024.

50 reprints are provided free for each article; additional copies may be obtained at cost in multiples of 50 .

The Pacific Journal of Mathematics is published monthly. Effective with Volume 16 the price per volume (3 numbers) is $\$ 8.00$; single issues, $\$ 3.00$. Special price for current issues to individual faculty members of supporting institutions and to individual members of the American Mathematical Society: $\$ 4.00$ per volume; single issues $\$ 1.50$. Back numbers are available.

Subscriptions, orders for back numbers, and changes of address should be sent to Pacific Journal of Mathematics, 103 Highland Boulevard, Berkeley, California, 94708.

PUBLISHED BY PACIFIC JOURNAL OF MATHEMATICS, A NON-PROFIT CORPORATION

Printed at Kokusai Bunken Insatsusha (International Academic Printing Co., Ltd.), 7-17, Fujimi 2-chome, Chiyoda-ku, Tokyo, Japan. 


\section{Pacific Journal of Mathematics}

\section{Vol. 37, No. $3 \quad$ March, 1971}

Mohammad Shafqat Ali and Marvin David Marcus, On the degree of the

minimal polynomial of a commutator operator ................ 561

Howard Anton and William J. Pervin, Integration on topological

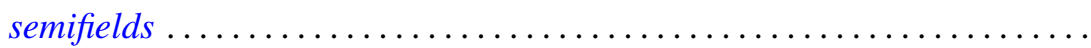

Martin Bartelt, Multipliers and operator algebras on bounded analytic

functions .................................... 575

Donald Earl Bennett, Aposyndetic properties of unicoherent continua ...... 585

James W. Bond, Lie algebras of genus one and genus two ............. 591

Mario Borelli, The cohomology of divisorial varieties ............... 617

Carlos R. Borges, How to recognize homeomorphisms and isometries ....... 625

J. C. Breckenridge, Burkill-Cesari integrals of quasi additive interval

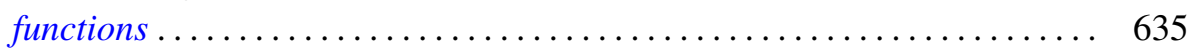

J. Csima, A class of counterexamples on permanents ................ 655

Carl Hanson Fitzgerald, Conformal mappings onto $\omega$-swirly domains . . . . . . 657

Newcomb Greenleaf, Analytic sheaves on Klein surfaces .............. 671

G. Goss and Giovanni Viglino, C-compact and functionally compact

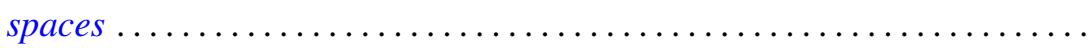

Charles Lemuel Hagopian, Arcwise connectivity of semi-aposyndetic plane

continua ..................................... 683

John Harris and Olga Higgins, Prime generators with parabolic limits ...

David Michael Henry, Stratifiable spaces, semi-stratifiable spaces, and their

relation through mappings .......................

Raymond D. Holmes, On contractive semigroups of mappings ........... 701

Joseph Edmund Kist and P. H. Maserick, BV-functions on semilattices ....... 711

Shûichirô Maeda, On point-free parallelism and Wilcox lattices ........... 725

Gary L. Musser, Linear semiprime $(p ; q)$ radicals ................. 749

William Charles Nemitz and Thomas Paul Whaley, Varieties of implicative

semilattices..................................... 759

Jaroslav Nešetřil, A congruence theorem for asymmetric trees ............ 771

Robert Anthony Nowlan, A study of $H$-spaces via left translations .......... 779

Gert Kjærgaard Pedersen, Atomic and diffuse functionals on a $C^{*}$-algebra ... 795

Tilak Raj Prabhakar, On the other set of the biorthogonal polynomials

suggested by the Laguerre polynomials...

801

Leland Edward Rogers, Mutually aposyndetic products of chainable

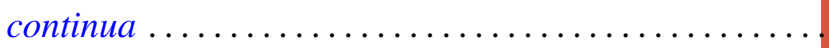

Frederick Stern, An estimate for Wiener integrals connected with squared

error in a Fourier series approximation.

Leonard Paul Sternbach, On k-shrinking and k-boundedly complete basic

sequences and quasi-reflexive spaces .................... 817

Pak-Ken Wong, Modular annihilator $A^{*}$-algebras ........ 